ARTICLE

Received 28 Jun 2013 | Accepted 21 Aug 2013 | Published 19 Sep 2013 DOl: 10.1038/ncomms3483

\title{
Atomic structure of amorphous shear bands in boron carbide
}

K. Madhav Reddy ${ }^{1}$, P. Liu ${ }^{1}$, A. Hirata', T. Fujita ${ }^{1} \&$ M.W. Chen ${ }^{1,2}$

Amorphous shear bands are the main deformation and failure mode of super-hard boron carbide subjected to shock loading and high pressures at room temperature. Nevertheless, the formation mechanisms of the amorphous shear bands remain a long-standing scientific curiosity mainly because of the lack of experimental structure information of the disordered shear bands, comprising light elements of carbon and boron only. Here we report the atomic structure of the amorphous shear bands in boron carbide characterized by state-of-the-art aberration-corrected transmission electron microscopy. Distorted icosahedra, displaced from the crystalline matrix, were observed in nano-sized amorphous bands that produce dislocation-like local shear strains. These experimental results provide direct experimental evidence that the formation of amorphous shear bands in boron carbide results from the disassembly of the icosahedra, driven by shear stresses.

\footnotetext{
${ }^{1}$ WPI Advanced Institute for Materials Research, Tohoku University, Sendai 980-8577, Japan. ${ }^{2}$ State Key Laboratory of Metal Matrix Composites, School of Materials Science and Engineering, Shanghai Jiao Tong University, Shanghai 200030, PR China. Correspondence and requests for materials should be addressed to M.W.C. (email: mwchen@wpi-aimr.tohoku.ac.jp).
} 
C ovalent boron carbide $\left(\mathrm{B}_{4} \mathrm{C}\right)$ is one of the hardest materials known in nature, after diamond and cubic boron nitride. The unusual bonding of 12-atom icosahedra at polar sites with a three-atom chain in the rhombohedra unit cell of $\mathrm{B}_{4} \mathrm{C}$ forms a large open internal space and strong covalent bonds, giving rise to the unique properties of being extremely hard ( $\sim 25$ $40 \mathrm{GPa}$ in hardness) but very light $\left(\sim 2.5 \mathrm{~g} \mathrm{~cm}^{-3} \text { in density }\right)^{1-7}$. Therefore, $\mathrm{B}_{4} \mathrm{C}$ has been an important structural ceramic for the applications where both high strength and lightweight are required $^{6,7}$. However, unlike other high performance ceramics, $\mathrm{B}_{4} \mathrm{C}$ displays an anomalous reduction in shear strength under pressures $^{8-11}$. High-resolution electron microscopy of shock-loaded fragments revealed that the formation of nano-scale amorphous shear bands is responsible for the dramatic loss of shear strength at high pressures ${ }^{12}$. Diamond anvil cell experiments suggested that the local amorphization of $\mathrm{B}_{4} \mathrm{C}$, associated with non-hydrostatic stress states, takes place during unloading from a pressure above a critical value of $\sim 26 \mathrm{GPa}^{13}$. In addition to shock loading and diamond anvil cell experiments, the amorphization has also been found when $\mathrm{B}_{4} \mathrm{C}$ is subjected to indentation and mechanical scratching ${ }^{14-17}$. These experimental observations demonstrated that the amorphous shear bands are the dominant deformation and failure modes of $\mathrm{B}_{4} \mathrm{C}$ at room temperature. On the basis of computer simulations and spectroscopic investigations, several controversial models have been proposed to explain the formation of amorphous shear bands in the super-hard material ${ }^{1,13,14,16-21}$. Nevertheless, the formation mechanisms of the amorphous shear bands still remain intensely debated owing to the lack of direct experimental observation with a sufficient spatial resolution to reveal the detailed structure of the amorphous bands.

In this study, we employed the state-of-the-art spherical aberration (Cs) corrected scanning transmission electron microscopy (STEM) to characterize the structure of the deformationinduced amorphous shear bands in $\mathrm{B}_{4} \mathrm{C}$. By utilizing the newly developed annular bright-field (ABF) STEM, which is advantageous for imaging light elements on atomic scale ${ }^{22,23}$, we provide direct evidence that the formation of amorphous shear bands results from the disassembly of the icosahedra in rhombohedral unit cells during shear deformation.

\section{Results}

Atomic structure of $\mathbf{B}_{\mathbf{4}} \mathbf{C}$. Although the lattice images of the rhombohedra $\mathrm{B}_{4} \mathrm{C}$ can be readily achieved by conventional high-resolution transmission electron microscopy $\left(\right.$ HRTEM) ${ }^{12,13}$, detailed atomic structure of $\mathrm{B}_{4} \mathrm{C}$, particularly 12-atom icosahedron and 3-atom chain in the unit cell, cannot be imaged because of the limited spatial resolution of the conventional HRTEM and the low contrast of the light elements of boron and carbon. In this study, we utilized ABF imaging technique using Cs-corrected STEM to achieve the ultrahigh spatial resolution of $\sim 0.1 \mathrm{~nm}$ for the light elements. Figure 1a shows the structural model of $\mathrm{B}_{4} \mathrm{C}$ projected along a [1011] direction, which consists of $\mathrm{B}_{11} \mathrm{C}$ icosahedra and linear $\mathrm{C}-$ $\mathrm{B}-\mathrm{C}$ atomic chains. The experimental ABF-STEM image (Fig. 1b) of crystalline $\mathrm{B}_{4} \mathrm{C}$ was taken from the thin region of a TEM specimen. Much more pronounced structural details, compared with the lattice images of HRTEM, can be observed. The ABFSTEM micrograph is consistent with the simulated ABF image as well (the inset of Fig. 1). The comparison between the experimental and simulated atomic images reveals the 12-atom icosahedra in the form of atomic rings and 3-atom chains as the dark lines linking the icosahedra. The ABF-STEM image is actually analogous to the direct projection of the structure model (Fig. 1a) along a same crystallographic direction. In fact, this is the first report of the atomic image of $\mathrm{B}_{4} \mathrm{C}$, and, therefore, $\mathrm{ABF}$ -
STEM is capable of detecting atomic-scale structure changes of $\mathrm{B}_{4} \mathrm{C}$, which could not be achieved before. In this study, we employed this advanced imaging technique to probe the local atomic structure of amorphous shear bands in deformed $\mathrm{B}_{4} \mathrm{C}$.

Raman spectroscopy of amorphous $\mathbf{B}_{4} \mathbf{C}$. In this study, the amorphous $\mathrm{B}_{4} \mathrm{C}$ was introduced by a nanoindentation experiment ${ }^{14,16,17,19}$. Figure 2 shows the representative Raman spectra acquired from the pristine and residual indented regions of $\mathrm{B}_{4} \mathrm{C}$. The spectrum of the pristine $\mathrm{B}_{4} \mathrm{C}$ displays characteristic Raman peaks at $270,320,481,531,728,830,1,000$ and $1,088 \mathrm{~cm}^{-1}$ assigned to crystalline $\mathrm{B}_{4} \mathrm{C}$ (refs 24,25), A weak band at $1,580 \mathrm{~cm}^{-1}$ has been attributed to the presence of a small fraction of graphitic carbon in sintered microcrystalline $\mathrm{B}_{4} \mathrm{C}$ (refs 7,16) (Fig. 2a). On indentation, the spectrum of the indented $\mathrm{B}_{4} \mathrm{C}$ reveals the most noticeable peak at $1,330 \mathrm{~cm}^{-1}$, followed by a shoulder peak at $1,520 \mathrm{~cm}^{-1}$ and a broad peak at $1,810 \mathrm{~cm}^{-1}$ (Fig. 2b). These characteristic Raman bands indicate the formation of amorphous $\mathrm{B}_{4} \mathrm{C}$ caused by the indentation, in agreement with previous observations of amorphous $\mathrm{B}_{4} \mathrm{C}$ in single and polycrystalline $\mathrm{B}_{4} \mathrm{C}$ ceramics ${ }^{14,16,17,19}$.

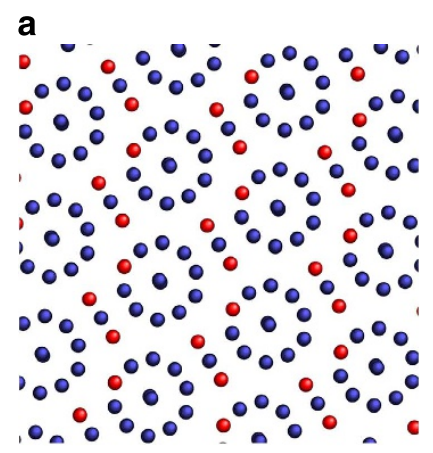

b

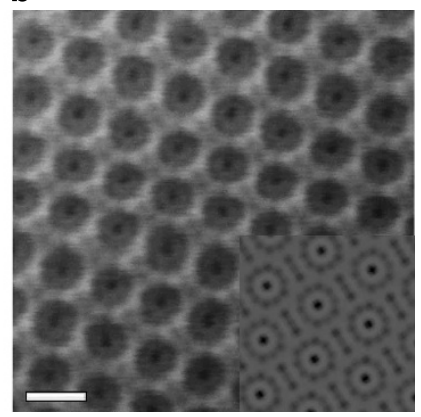

Figure 1 | Structure model and atomic image of $\mathbf{B}_{\mathbf{4}} \mathbf{C}$. (a) Projected structure model of the $\mathrm{B}_{4} \mathrm{C}$ along the [10101] direction. Boron in blue and carbon in red colour. (b) Experimental ABF-STEM image of $\mathrm{B}_{4} \mathrm{C}$ along [10111]. Scale bar, $5 \AA$. The simulated ABF-STEM image is inserted at the bottom side.

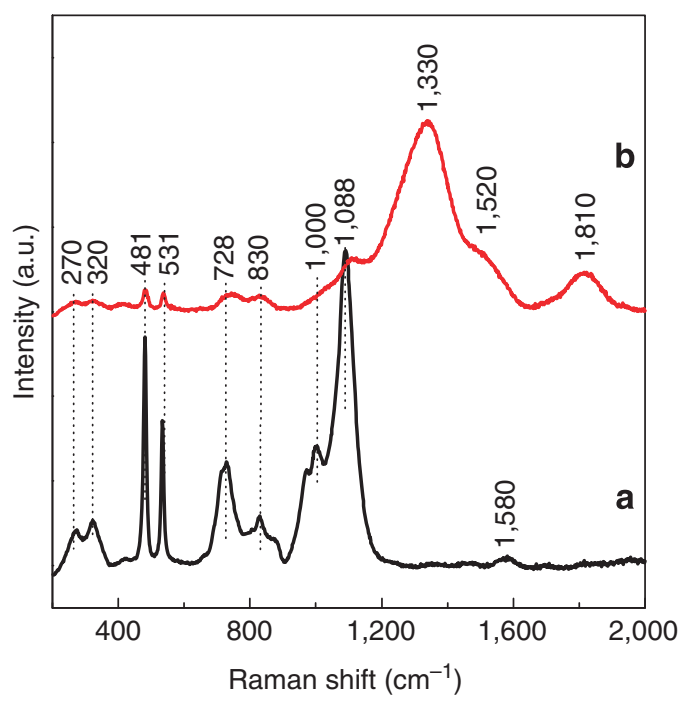

Figure 2 | Raman spectra of amorphous and crystalline $\mathbf{B}_{\mathbf{4}} \mathbf{C}$. (a) Representative Raman spectrum of pristine $\mathrm{B}_{4} \mathrm{C}$; and (b) Raman spectrum taken from a residual indentation of microcrystalline $\mathrm{B}_{4} \mathrm{C}$ with $514 \mathrm{~nm}$ argon ion laser excitation. 
ABF-STEM of amorphous shear bands. To illustrate the structure of the indentation-induced amorphous $\mathrm{B}_{4} \mathrm{C}$, the cross-sectional TEM specimen was sliced from a residual indent using a focused ion beam milling (FIB) technique ${ }^{26,27}$. The detailed process has been illustrated in Fig. 3 and described in the Methods section. Before cutting, an indentation region with the crystallographic direction [10 $\overline{1} 1]$ was selected from the polycrystalline sample by using electron backscattered diffraction. Figure 4 shows the STEM images of the indentation region of $\mathrm{B}_{4} \mathrm{C}$. Shear bands and micro-cracks, marked by the arrowheads, can be seen from the low magnification bright- and dark-field STEM images along the [10 $\overline{1} 1]$ crystallographic direction (Fig. 4a,b), suggesting that extensive plastic deformation and damage take place underneath the indenter. In general, the micro-cracks show high contrast owing to complete separation by an open gap between two sides. Shear bands have much low contrast and usually have a width of $\sim 2-3 \mathrm{~nm}$ and a length of $\sim 100-200 \mathrm{~nm}$ (Fig. 4c). The zoom-in ABF-STEM image shows the loss of periodic crystalline structure within a shear band but voids and open gaps cannot be seen (Fig. 4d). The fast Fourier transform (FFT) pattern taken from the shear band displays a diffuse halo ring, confirming the occurrence of amorphization within the shear band (Fig. $4 \mathrm{~d}$, inset B). In the crystalline substrate, the periodic icosahedron network can be well illustrated by the ABF-STEM image, implying that the sample fabricated by FIB is sufficiently thin to retain the high spatial resolution of ABFSTEM. The FFT patterns of the crystalline matrix display only the rhombohedral symmetry (Fig. 4d, inset A and C). Therefore, except for shear bands and micro-cracks, the crystalline $\mathrm{B}_{4} \mathrm{C}$ matrix does not experience any structural transformation during the heavy indentation deformation. From the FFT patterns of the crystalline matrix, it can be estimated that there is $\sim 2^{\circ}$ angular mismatch of the crystals between the two sides of the amorphous a

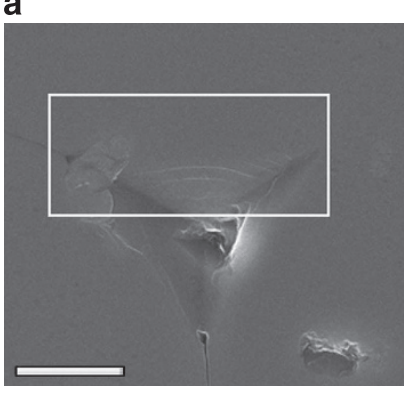

C

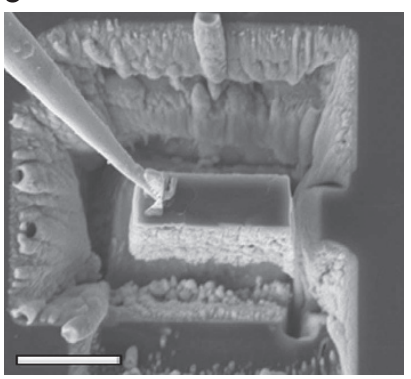

b

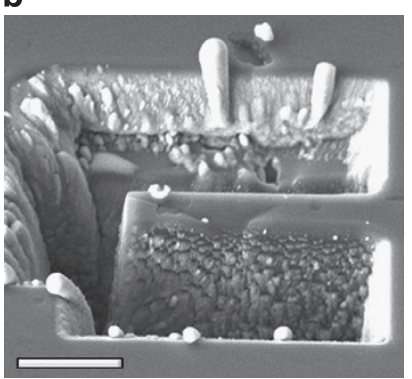

d

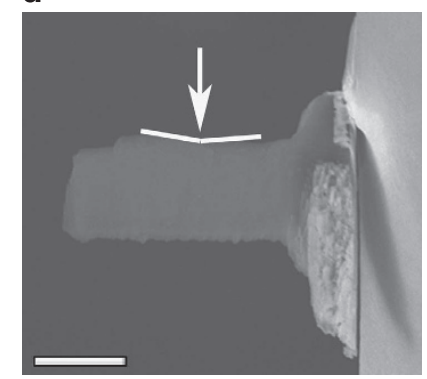

Figure 3 | Cross-sectional TEM sample preparation by FIB milling.

(a) The selected region from the indentation impression (shown in white box) with the crystallographic direction of [1011], estimated by electron backscatter diffraction. Scale bar, $5 \mu \mathrm{m}$. (b) Large trenches made around the selected region by FIB cutting. Scale bar, $10 \mu \mathrm{m}$. (c) A wedge-shaped TEM foil extracted by a microprobe. Scale bar, $10 \mu \mathrm{m}$. (d) Side view of the wedge-shaped specimen attached on the copper mesh by tungsten deposition (arrow showing indented impression). Scale bar, $5 \mu \mathrm{m}$. band, which is similar to the previous observations of amorphous shear bands in shock-loaded and diamond-anvil-cell-tested $\mathrm{B}_{4} \mathrm{C}$ (refs 12,13).

The detailed structure of the amorphous shear band shown in Fig. $4 \mathrm{~d}$ is viewed from the direction close to [10 $\overline{1} 1]$ of the crystal matrix. Because of the elastic strain field around the amorphous shear band, there is an orientation mismatch between the crystals residing at both sides of the amorphous band, as shown by the FFT patterns in Fig. 4d. Thus, it is difficult to align both crystals at their exact zone orientations simultaneously. The sample was first tilted to the orientation showing the sharpest crystal/ amorphous interfaces, and, meanwhile, the crystal structure can be identified from both sides of the $\sim 2 \mathrm{~nm}$ amorphous band (Fig. 5a). Deformed icosahedra with an imperfect ring shape are observed at the interface between the amorphous band and the crystal matrix, as shown in white circles (Fig. 5a). Although some icosahedra are significantly displaced from the lattice sites, others are hardly displaced, illustrating the gradual structure change from crystal to amorphous at the subnanoscale. As both crystals at the two sides of the amorphous band are not aligned at their exact zone orientation along this viewing direction, the defocus is set to image the crystal matrix and the structure of the amorphous band may be partially concealed as shown in Fig. 5a. To resolve the ambiguity of the amorphous band, the top-side crystal is perfectly aligned to [10 $\overline{1} 1]$ projection (Fig. 5b). From this on-axis image, we can see that the icosahedral clusters are a

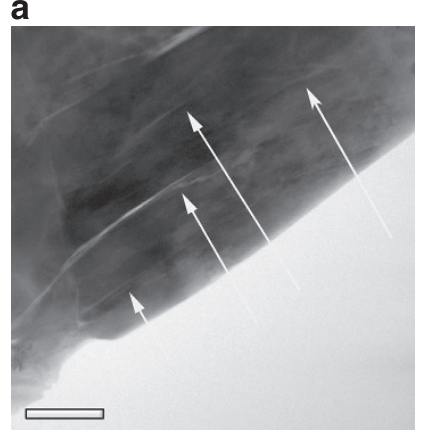

C

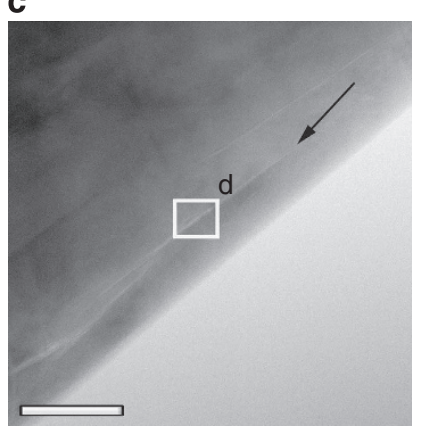

b

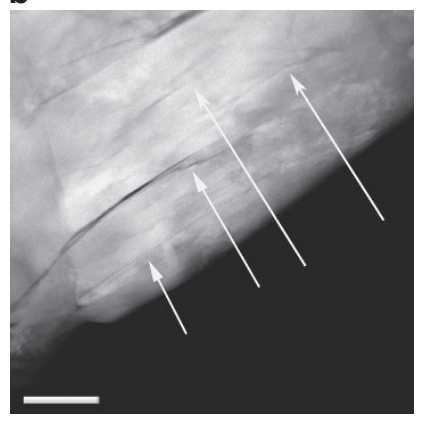

d

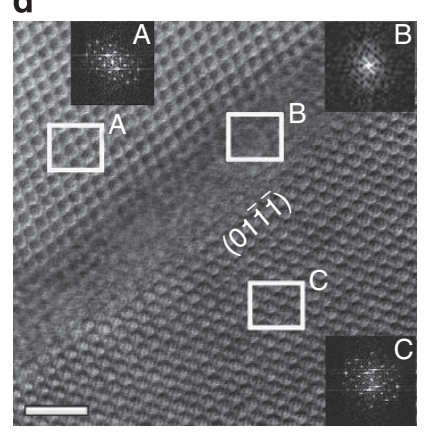

Figure 4 | Amorphous shear bands within the deformed area. (a) Low magnification bright-field and (b) dark-field STEM images of the deformed region. The shear bands and micro-cracks are indicated with arrowheads. Scale bar, $500 \mathrm{~nm}$. (c) Bright-field STEM image of a zoom-in shear band with a length of $\sim 200 \mathrm{~nm}$. The black arrowhead shows the tip of the shear band. Scale bar, $50 \mathrm{~nm}$. (d) ABF-STEM image of the shear band taken from the white-box region in (c). The high-resolution STEM image shows the amorphous structure of the shear band with a width of $\sim 2 \mathrm{~nm}$ along the [1011] crystallographic direction. Inset FFT patterns demonstrate the amorphous nature of the shear band. The slight mismatch in the orientation between the top and the bottom crystals can also be seen from the corresponding FFT patterns. Scale bar, $2 \mathrm{~nm}$. 

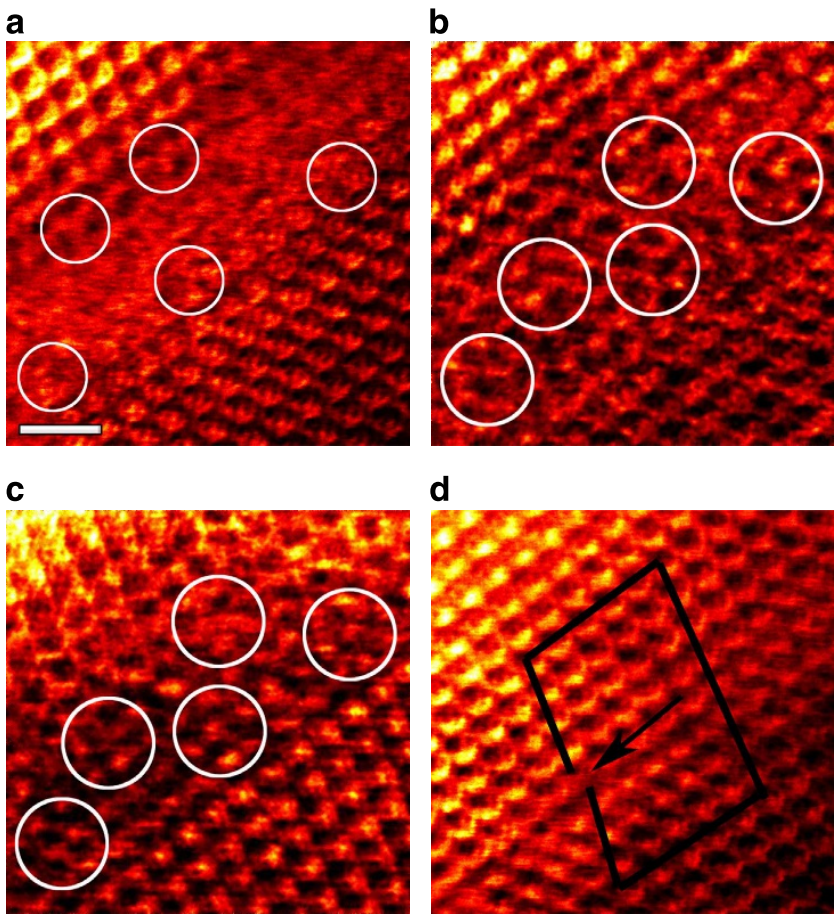

Figure 5 | ABF-STEM images of an amorphous shear band. (a) The image taken from slightly off-axis orientation from the [10111] $B_{4} C$ crystalline matrix. The displaced icosahedra at the amorphous/crystal interface are marked in white colour circles. (b,c) Retained icosahedra in the amorphous shear band imaged by on-axis ABF-STEM on either side of the crystal. The randomly distributed icosahedra in the amorphous band are denoted with white circles. (d) Shear displacement at the tip of the amorphous shear band. All images scale bar, $1 \mathrm{~nm}$.

well unveiled, which are randomly distributed in the amorphous band. Moreover, the icosahedra loss their regular shape (shown in white circles). To further clarify the amorphous $\mathrm{B}_{4} \mathrm{C}$ structure, we have adjusted the bottom side (Fig. 5a) to the exact [1011] zone direction (Fig. 5c). From this view, we can readily detect the dangled and distorted icosahedral clusters in amorphous $\mathrm{B}_{4} \mathrm{C}$ region shown in white circles (Fig. $5 \mathrm{c}$ ). Although the pronounced icosahedra can be recognized in the amorphous zone, their number density is apparently lower than that of the crystal matrix. This may be due to some icosahedra directly collapsing during the amorphization or tilting to orientations that cannot be imaged by ABF-STEM.

Figure $5 \mathrm{~d}$ is the ABF-STEM image taken from the tip region of the amorphous band, marked by the black arrowhead in Fig. 4c. The Burgers loop reveals the obvious lattice displacement along the amorphous band. The accumulated lattice strain at the tip of the amorphous band is analogous to a partial dislocation with a Burgers vector parallel to the shear band direction. Therefore, the amorphization of $\mathrm{B}_{4} \mathrm{C}$ gives rise to a shear displacement, equivalent to dislocation deformation in ductile crystals. On the other hand, this observation also demonstrates that the amorphization of $\mathrm{B}_{4} \mathrm{C}$ results from shear deformation.

\section{Discussion}

It is interesting to note that the amorphous shear bands, revealed by the ABF-STEM images, are along the crystal plane (011̄1) that is comprised of aligned three-atom chains (Fig. 6). However, this plane is not the favourable dislocation slip plane of the rhombohedra $\mathrm{B}_{4} \mathrm{C}$ because the magnitude of the Burgers vector

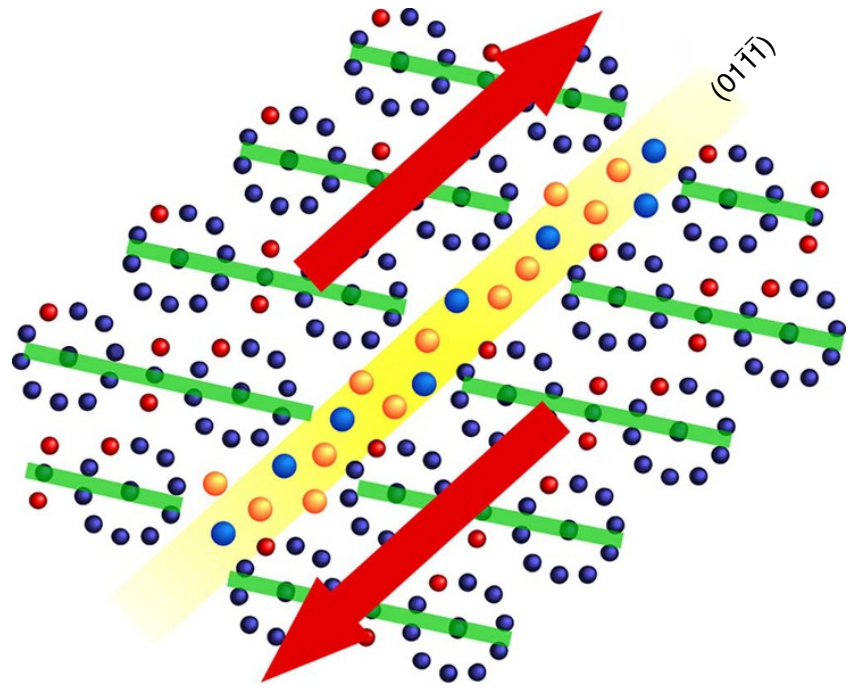

Figure 6 | Schematic of $B_{\mathbf{4}} C$ amorphization by shear sliding along the plane. Shear-induced amorphization preferentially takes place along the $\mathrm{B}_{4} \mathrm{C}(01 \overline{1} \overline{1})$ plane that is comprises three-atom chains.

of dislocations on this plane is too large. Thus, dislocations and stacking faults cannot be formed on this plane under shear stress. Compared to the spherical icosahedra with closed atomic bonding, the three-atom chains are easier to deform (bending) and break when applied forces are not loaded along the chain direction ${ }^{13,20}$ Therefore, shear deformation preferentially takes place along this plane by the deformation and debonding of the three-atom chains when regular dislocation slip cannot be activated due to high Peierls stress at low temperatures. The occurrence of shearing along this plane may also be stimulated by the accumulation of thermal equilibrium point defects in the atomic chains ${ }^{28}$, which leads to the weakening of the chain structure. Likewise, multiple polyhedra of the chain structure $(\mathrm{C}-\mathrm{B}-\mathrm{C}, \mathrm{C}-\mathrm{C}-\mathrm{C}, \mathrm{C}-\mathrm{C}-\mathrm{B}$ and so on $)^{20}$, together with the point defects (vacancies) in the chains, make this slip plane not have good long-range translational symmetry, and, hence, favourably form structure disorder when slip takes place. Moreover, the two identical debonding sites at the three-atom chains can also easily induce structure disorder when shear deformation takes place along this plane.

In summary, we have directly imaged the structure of amorphous shear bands in $\mathrm{B}_{4} \mathrm{C}$ by using the state-of-the-art ABF-STEM. The atomic-scale observations demonstrate the existence of retained icosahedra in nano-scale amorphous bands. Combining with crystallographic analysis, this study provides compelling experimental evidence on the underlying mechanisms of shear-induced amorphization of $\mathrm{B}_{4} \mathrm{C}$.

\section{Methods}

Material fabrication and nanoindentation experiment. Fully dense microcrystalline boron carbide ceramic was consolidated by a hot pressing $\operatorname{method}^{7}$. The grain size of the sample ranges from $3-15 \mu \mathrm{m}$. A mirror-finish surface was prepared by careful polishing using diamond films. A series of indentations at a constant load of $2 \mathrm{~N}$ were performed with a dynamic ultra-micro-hardness tester (Shimadzu W201S) equipped with a Berkovich indenter. The mean hardness of the polycrystalline $\mathrm{B}_{4} \mathrm{C}$ was measured to be $\sim 27 \mathrm{GPa}$ and these indents were examined using a micro-Raman spectrometer (Renishaw, UK) with a $514 \mathrm{~nm}$ laser. The diameter of the laser spot was $\sim 1 \mu \mathrm{m}$, which is $\sim 4$ to 5 times smaller than the dimension of residual impressions.

TEM specimen preparation and characterization. The cross-sectional TEM specimens of the indented $\mathrm{B}_{4} \mathrm{C}$ were prepared by a lift-out technique using a FIB system (JEOL JIB-4600F) (Fig. 3). The indented $\mathrm{B}_{4} \mathrm{C}$ specimen was tilted to an angle of $\sim 53^{\circ}$ in the FIB system, and a large trench was made on either side of indented region using a high $\mathrm{Ga}^{+}$beam current with the accelerated 
voltage of $30 \mathrm{kV}$ (Fig. 3b). After removing the remaining residual portion, the specimen was tilted back to its original position and a microprobe was attached on the top surface of the indent using tungsten deposition (Fig. 3c). The TEM foil was subsequently lifted out and attached onto a copper grid by tungsten deposition, followed by removal of microprobe tip from the TEM foil by $\mathrm{Ga}^{+}$beam cutting. Finally, the sample is thinned down to a thickness $<50 \mathrm{~nm}$ by a low-energy ion beam (Fig. 3d). Before the TEM observations, the TEM sample was further gently milled by a Fishone 1040 Nanomill system to remove the damage layer on the sample surface without altering the specimen chemistry and structure. The microstructure of deformed samples was characterized with a TEM (TEM, JEOL JEM-2100F) with double Cs correctors. The ABF-STEM images were recorded by Gatan DF detector with the detector angle of $\sim 11-23 \mathrm{mrad}$ and point-to-point resolution $0.1 \mathrm{~nm}$. ABF-STEM image simulations were performed by using a software package of Win HREM (HREM Research Inc)

\section{References}

1. Domnich, V., Reynaud, S., Haber, R. A. \& Chhowalla, M. Boron carbide: structure, properties, and stability under stress. J. Am. Ceram. Soc. 94, 3605-3628 (2011).

2. Mauri, F., Vast, N. \& Pickard, C. J. Atomic structure of icosahedral $\mathrm{B}_{4} \mathrm{C}$ boron carbide from a first principles analysis of NMR spectra. Phys. Rev. Lett. 87, 085506 (2001).

3. Kwei, G. H. \& Morosin, B. Structures of the boron-rich boron carbides from neutron powder diffraction: Implications for the nature of the inter-icosahedral chains. J. Phys. Chem. 100, 8031-8039 (1996).

4. Nelmes, R. J., Loveday, J. S., Wilson, R. M. \& Marshall, W. G. Observation of inverted-molecular compression in boron carbide. Phys. Rev. Lett. 74, 2268-2271 (1995).

5. Aselage, T. L., Emin, D., McCready, S. S. \& Duncan, R. V. Large enhancement of boron carbides' seebeck coefficients through vibrational softening. Phys. Rev. Lett. 81, 2316-2319 (1998).

6. Madhav Reddy, K. et al. Enhanced mechanical properties of nanocrystalline boron carbide by nanoporosity and interface phases. Nat. Commun. 3, 1052 (2012).

7. Chen, M. W., McCauley, J. W., Lasalvia, J. C. \& Hemker, K. J. Microstructural characterization of commercial hot-pressed boron carbide ceramics. J. Am. Ceram. Soc. 88, 1935-1942 (2005).

8. Grady, D. E. Shock-wave compression of brittle solids. Mech. Mater. 29, 181-203 (1998).

9. Dandekar, D. P. Shock response of boron carbide, US Army Research Laboratory Report No. ARL-TR-2456 (2001).

10. Vogler, T. J., Reinhart, W. D. \& Chhabildas, L. C. Dynamic behaviour of boron carbide. J. Appl. Phys. 95, 4173-4183 (2004).

11. Bourne, N. K. et al. On the Hugoniot elastic limit in polycrystalline alumina. J. Appl. Phys. 102, 073514 (2007).

12. Chen, M. W., McCauley, J. W. \& Hemker, K. J. Shock-induced amorphization in boron carbide. Science 299, 1563-1566 (2003).

13. Yan, X. Q. et al. Depressurization amorphization of single crystal boron carbide. Phys. Rev. Lett. 102, 075505 (2009).

14. Ge, D. et al. Structural damage in boron carbide under contact loading. Acta Mater. 52, 3921-3927 (2004).

15. Chen, M. W. \& McCauley, J. W. Mechanical scratching induced phase transitions and reactions of boron carbide. J. Appl. Phys. 100, 123517 (2006).

16. Yan, X. Q., Li, W. J., Goto, T. \& Chen, M. W. Raman spectroscopy of pressure-induced amorphous boron carbide. Appl. Phys. Lett. 88, 131905 (2006).
17. Subhash, G. et al. Characterization of the 3-D amorphized zone beneath a Vickers indentation in boron carbide using Raman spectroscopy. Acta Mater. 61, 3888-3896 (2013).

18. Guo, J. J., Zhang, L., Fujita, T., Goto, T. \& Chen, M. W. Pressure induced depolarization and resonance in Raman scattering of single crystalline boron carbide. Phys. Rev. B 81, 060102 (2010).

19. Ghosh, D., Subhash, G., Lee, C. H. \& Yap, Y. K. Strain induced formation of carbon and boron clusters in boron carbide during dynamic indentation. Appl. Phys. Lett. 91, 061910 (2007).

20. Fanchini, G., McCauley, J. W. \& Chhowalla, M. Behavior of disordered boron carbide under stress. Phys. Rev. Lett. 97, 035502 (2006).

21. Aryal, S., Rulis, P. \& Ching, W. Y. Mechanism for amorphization of boron carbide $\mathrm{B}_{4} \mathrm{C}$ under uniaxial compression. Phys. Rev. B 84, 184112 (2011).

22. Okunishi, E. et al. Visualization of light elements at ultrahigh resolution by STEM annular bright field microscopy. Microsc. Microanal. 15, 164-165 (2009).

23. Ishikawa, R. et al. Direct imaging of hydrogen-atom columns in a crystal by annular bright-field electron microscopy. Nature Mater. 10, 278-281 (2011).

24. Werheit, H. et al. IR-Active phonons and structure elements of isotopeenriched boron carbide. J. Solid State Chem. 154, 79-86 (2000).

25. Lazzari, R., Vast, V., Besson, J. M., Baroni, S. \& Corso, D. Atomic structure and vibrational properties of icosahedral $\mathrm{B}_{4} \mathrm{C}$ boron carbide. Phys. Rev. Lett. 83 , 3230-3233 (1999).

26. Guo, J. J. et al. Nanoindentation characterization of deformation and failure of aluminium oxynitride. Acta Mater. 59, 1671-1679 (2011).

27. Giannuzzi, L. A. \& Stevie, F. A. A review of focused ion beam milling techniques for TEM specimen preparation. Micron 30, 197-204 (1999).

28. Raucoules, R., Vast, N., Betranhandy, E. \& Sjakste, J. Mechanical properties of icosahedral boron carbide explained from first principles. Phys. Rev. B 84, $014112(2011)$.

\section{Acknowledgements}

This work is sponsored by JST-CREST 'Phase Interface Science for Highly Efficient Energy Utilization' and 'World Premier International (WPI) Center Initiative for Atoms, Molecules and Materials,' MEXT, Japan; and National Natural Science Foundation of China (51271113). T.F. is partially supported by JST-PRESTO program.

\section{Author contributions}

M.W.C. designed and supervised this study. K.M.R. conducted the mechanical tests and ABF-STEM characterization. P.L., A.H. and T.F. contributed to the STEM study. A.H. performed ABF-STEM image simulation. M.W.C. and K.M.R. wrote the paper. All the authors commented on the manuscript writing.

\section{Additional information}

Competing financial interests: The authors declare no competing financial interests

Reprints and permission information is available online at http://npg.nature.com/ reprintsandpermissions/

How to cite this article: Reddy, K.M. et al. Atomic structure of amorphous shear bands in boron carbide. Nat. Commun. 4:2483 doi: 10.1038/ncomms3483 (2013). 